\title{
Un compás que hace cuadrados: La escritura y la lectura en el currículum de séptimo año en Costa Rica
}

A square drawing compass: writing and reading in the first year of high school in Costa Rica

\section{Volumen 18, Número 1 \\ Enero-Abril}

pp. 1-29

Catalina Ramírez Molina

Revista indizada en REDALYC, SCIELO

Revista distribuida en las bases de datos:

LATINDEX, DOAJ, REDIB, IRESIE, CLASE, DIALNET, SHERPA/ROMEO, QUALIS-CAPES, MIAR

Revista registrada en los directorios:

ULRICH'S, REDIE, RINACE, OEI, MAESTROTECA, PREAL, CLACSO 


\title{
Un compás que hace cuadrados: La escritura y la lectura en el currículum de séptimo año en Costa Rica
}

\author{
A square drawing compass: writing and reading in the first year of high school \\ in Costa Rica
}

\begin{abstract}
Catalina Ramírez Molina ${ }^{1}$
Resumen: El presente artículo muestra los resultados de un análisis en profundidad del documento curricular, mediante el método de redes sistémicas, de las áreas de lectura y escritura del Programa de estudios de séptimo año en Costa Rica. El propósito es conocer en primer lugar, qué contempla el texto curricular en estas dos áreas $y$ en segundo lugar, ofrecer un contraste entre los resultados del análisis y las nuevas concepciones didácticas para la enseñanza de la lengua y la literatura. El análisis realizado revela una propuesta curricular desmantelada de conocimientos didácticos, que oriente las prácticas de escritura y lectura en secundaria. Por lo tanto, se proponen algunos cambios para una propuesta curricular que permita a la persona docente planificar una programación coherente que asegure a sus estudiantes la apropiación de una alfabetización en sintonía con el desarrollo de competencias de lectura y escritura necesarias para vivir hoy.
\end{abstract}

Palabras clave: Educación secundaria, currículum, escritura, lectura.

Abstract: This article presents the results of an in-depth analysis of the curricular document of reading and writing areas of the first year of high school Program of Studies in Costa Rica, using the systemic networks approach. The purpose is to know firstly, the contents of the curricular text regarding these two areas. Secondly, there is a contrast between the results of the analysis and the new didactic conceptions for the teaching of language and literature. The analysis reveals a curricular proposal lacking of didactic knowledge, which guides the practices of writing and reading in secondary school. Therefore, some changes are suggested as a curricular proposal that allows teachers to plan a coherent program that assures their students the appropriation of a literacy in harmony with the development of reading and writing skills necessary to live today.

Key Words: High school education, curriculum, writing, reading.

${ }^{1}$ Docente en la Enseñanza del Castellano y la Literatura en la Universidad de Costa Rica.

Dirección electrónica: ramrezmolinac@gmail.com

Artículo recibido: 28 de abril, 2017

Enviado a corrección: 13 de junio, 2017

Aprobado: 18 de setiembre, 2017 


\section{Introducción}

Compartir en el presente artículo los resultados del análisis del documento curricular en las áreas de escritura y lectura nace de dos experiencias. La primera surgió hace varios años atrás con mis estudiantes universitarios al acercamos al currículum para la enseñanza del Español de la secundaria. En tiempos de conversación y reflexión, el estudiantado universitario expresaba tensiones y contradicciones al enfrentar el texto curricular de séptimo año, y llegamos a una conclusión: de manera metafórica, el currículum de español no es más que un muñeco de trapo empolvado que cada cuatro u ocho años se recompone con atuendos de moda y maquillaje para salir a escena. El público aplaude, pero los que estamos tras bastidores, planeando las escenas, sabemos que en cualquier momento un dedo, un ojo o una pierna caerá.

Con la descripción de esta inquietante imagen comenzó el interés por conocer de qué manera estaban organizadas y cómo se concebían las áreas de lectura y escritura en el documento curricular, y a este interés se unió otra imagen (segunda experiencia), creada por Guiseppe Calarusso (2014), un manipulador de la fotografía. Su proyecto, titulado Improbabilitá ('improbabilidad'), muestra fotografías surrealistas de objetos cotidianos pero con un giro: el artista los modifica y los convierte en objetos carentes de funcionalidad. La obra de Calarusso pretende hacer reflexionar sobre lo asumidas que están las formas de todos esos objetos de uso cotidiano. En una de las fotografías se retrata una hoja blanca de papel en la que se muestra un cuadrado trazado por un compás. Esta imagen, que es simple solo en apariencia, encierra una gran complejidad, pues sabemos que el compás es un objeto diseñado para crear una figura geométrica perfecta: el círculo, pero en la imagen lo que se muestra es un cuadrado. Al ver esta fotografía, comprendí que exactamente eso que lograba visualizar, es lo que muchos docentes experimentamos en el ámbito educativo, específicamente en el didáctico y curricular para la enseñanza de la lengua y la literatura.

Por muchos años, en Costa Rica, el currículum de español de secundaria ha sido un objeto cotidiano, semejante al compás. Todos los docentes asumimos que existe y cumple una función: trazar círculos, y los docentes nos encargamos de hacerlo girar en la dirección adecuada, ya que las tareas de enseñar y aprender requieren de un tratamiento cíclico en el que se integran experiencias, conocimientos y habilidades. No obstante, varios investigadores en la materia (Arias, Ruíz y Vargas, 2013; Casaca, 2009; Gallardo, 2006; Méndez, 2000; Murillo, 2009; Ramírez, 2006; Ovares, Alfaro, Rojas y Mora, 1986; Sánchez, 2005), muestran que el compás en lugar de trazar círculos, hace cuadrados. 
Ofrecer una respuesta al porqué de este fenómeno implicaba comprenderlo desde el origen. Partir del currículum era una tarea ineludible, pues en él se establecen las nociones didácticas relativas a la escritura y la lectura: qué enfoques de enseñanza las orientan; cuáles corrientes teóricas las sustentan; cómo se plantea su aprendizaje; qué roles son asignados al docente y al estudiante; qué se espera lograr con esa enseñanza; cuáles son los componentes curriculares (objetivos, contenidos, actividades, valores, evaluación), y cómo se estructuran y organizan. Revelar, en el currículum, la dinámica, las conexiones y las desconexiones de esta información requería de una lectura profunda y exhaustiva que permitiera mostrar cómo se plantea su enseñanza y aprendizaje.

Ante estas experiencias, el interés de la presente aportación es mostrar un acercamiento a la geografía de las áreas de escritura y lectura en el Programa de estudios de séptimo año para revelar lo que encierra, describirla, analizarla, con detenimiento crítico y perspectiva didáctica. Para llevar a cabo estas tareas, se realizó un trabajo cualitativo que permitiera analizar el documento curricular, contrastar los resultados del análisis con los avances investigativos en el campo de la didáctica de la lengua y la literatura y establecer posibles giros de cambio para una propuesta curricular y didáctica coherente.

\section{Referente teórico del estudio}

El sustento teórico de la investigación descansa en tres ejes temáticos: 1 . El currículum 2. La enseñanza de la escritura 3 . La enseñanza de la lectura. Estos tres ejes son campos vastos y complejos, por lo que se realiza una selección y delimitación de aspectos pertinentes de cada uno. En el caso del primer eje temático, se presenta una breve conceptualización acerca de lo que entenderemos por currículum, cuáles son sus componentes, qué funciones deben cumplir y por qué este texto es indispensable en la actuación docente. En el segundo y tercer eje temáticos: La enseñanza de la escritura y la enseñanza de la lectura, se intenta bosquejar cómo se han entendido estas prácticas tradicionalmente y de qué forma (a través de diversos estudios provenientes del campo de la didáctica de la lengua y la literatura) se abre un nuevo y dinámico panorama relativo a los conocimientos que deben considerarse y de qué manera estos cambios paradigmáticos obligan a revisar y analizar las formas en que los objetos de enseñanza (en nuestro caso, la escritura y la lectura) se plantean en el currículum oficial de secundaria en Costa Rica. 


\subsection{Definición moderna del currículum}

Las definiciones modernas del currículum son numerosas y siempre sujetas a un debate vivo e inacabado, esto es bueno porque ya hemos interiorizado que el acto educativo es fluctuante e impredecible. En este punto nos referimos al enfoque mixto propuesto por Akker, Fasoglio y Mulder (2010), en el que se integran diversas tendencias y enfoques de desarrollo en el campo de la educación y se genera un distanciamiento en cuanto a adscribirse a un solo enfoque según la teoría curricular, es decir, en lugar de pensar en el currículum desde un único modelo, se intenta llevar a cabo una mezcla de tendencias y enfoques articulados y contextualizados.

El enfoque mixto contempla el pragmatismo, los prototipos, la comunicación y el desarrollo profesional. El contexto práctico y sus usuarios están a la vanguardia del diseño curricular y su promulgación. Este tipo de procesos, acompañado de un estilo comunicativo relacional, es deseable para llegar a los inevitables compromisos entre actores con diferentes roles e intereses y para crear una coherencia externa entre todas las partes involucradas (Akker et al., 2010, pp. 12-13).

A través de este enfoque mixto, no solo se considera el currículum como la expresión y concreción del plan cultural en la institución escolar, sino que el currículum intentado (el texto curricular) ofrece una coherencia abierta y flexible para que la persona docente pueda ejercer un currículum en el que pueda analizar el conocimiento teórico disponible, y utilice el conocimiento que él mismo obtiene de la reflexión sobre su práctica, y su experiencia como docente (Schön, 1998, p. 280). Este nuevo paradigma, para entender los alcances del currículum, alimenta la noción del texto curricular como el primer espacio que reúne y estructura el conocimiento y, además, orienta sobre lo que ha de ser impartido, reproducido en los estudiantes bajo el orden de un determinado formato.

Aunque, en apariencia, este estudio se centró en la parte tradicional del currículum, que es el texto curricular, consideramos de enorme trascendencia partir de él, pues a través de este primer paso, tocamos el terreno de la nueva línea para entender el currículum, que es comprenderlo como instrumento que permite la deliberación y que si representa el punto de partida de las prácticas docentes, pueda ser un espacio para reflexionar sobre los supuestos, contenidos y representaciones de prácticas de la escritura y la lectura que favorezcan las reformulaciones. Por lo tanto, en palabras de Linuesa (2010) ¿Qué toca al currículum? Prever y representar la acción. 


\subsection{Componentes curriculares}

El diseño curricular, según Walker (1990), incluye tres grandes elementos de planificación: el contenido, la finalidad y la organización del aprendizaje. Desde esta perspectiva, el currículum elige los saberes y las competencias que deben ser desarrollados por el estudiantado en el sistema escolar. Desde el enfoque tradicional del currículum, a través de estos componentes, se organiza la enseñanza que deben impartir los docentes y el aprendizaje que deben alcanzar los estudiantes. Con el propósito de analizar la propuesta curricular para la enseñanza de la escritura y la lectura de secundaria en Costa Rica, fue necesario comprender cuáles componentes curriculares se planteaban, cómo se organizaban y cuáles son sus relaciones y funciones. Para ello, en este apartado se detalla brevemente qué se entiende en el estudio — desde la teoría curricular- por objetivos de aprendizaje, contenidos, actividades de aprendizaje y evaluación.

\subsubsection{Objetivos de enseñanza, contenidos, actividades de aprendizaje y evaluación}

Tyler (1973) señala que la formulación de objetivos debe responder a las preguntas: ¿para qué hacerlo?, ¿hacia cuáles metas se dirigen para aprender? Por lo tanto, deben enunciarse describiendo los cambios que se espera lograr en las personas estudiantes, no lo que harán los profesores. En este sentido, los objetivos son cambios que el sujeto consigue como consecuencia del aprendizaje y deben relacionarse con cada una de las actividades planteadas.

La principal crítica a la formulación de objetivos tiene que ver con las clasificaciones taxonómicas propias del modelo técnico. Linuesa (2010) explica que para facilitar la selección de objetivos surgieron las denominadas taxonomías de Bloom (1973) y Gagné (1979); listas de tipos de objetivos, si bien, referidas a tipos de aprendizaje. En un momento, la pedagogía por objetivos permitía que todos trabajaran una estructura común desde una aparente sencillez. Gimeno (1982) señala que

esa sencillez se convirtió en un artilugio debido a que en la realidad se produjo más tecnicismo que cientificidad, la tendencia fue confundir fines, resultados, conductas y objetivos. Las taxonomías presentan una visión sumativa del conocimiento, no contemplan la transferencia ni el descubrimiento en el aprendizaje y se sustentan en bases psicológicas irrelevantes, que hoy son insostenibles. (p. 276) 
Desde esta lectura, las taxonomías se acercaban más a una educación reproductora que constructiva, su dificultad para planificar determinadas áreas de conocimiento fue notable. Debido a este cuestionamiento, muchos currículos han decidido pasar de una pedagogía por objetivos a una pedagogía por competencias; no obstante, Gimeno (2010) señala al respecto, que en la nueva tendencia de sustituir una pedagogía por otra se corre el riesgo de convertir de nuevo las competencias —al igual que antes los objetivos - en instrumentos normativos, fines, contenidos y guías para elegir procedimientos y propuestas para la evaluación (p. 277). Este dilema hace que el concepto de competencias sea excesivamente amplio, en el que la precisión y la mediación se convierten en valores absolutos y la educación no es un producto, sino un proceso en el que se hace complejo establecer medidas concretas.

En este sentido, no solo es necesario conocer los límites epistémicos del concepto de competencia, dada la dificultad para universalizarlo, sino también los intereses que lleva consigo y que se proyectan en el uso que se haga de él. No solo debe ser una nueva moda, sino un cambio epistemológico en su carácter (Barnett, 2001, p.108). Por lo tanto, la competencia debe poseer tres rasgos distintivos: la transversalidad, la transferencia y la progresión, no solo la tenencia de saberes o el saber hacer, sino también el saber actuar.

Por otro lado, los debates sobre los contenidos del currículum han sido más que numerosos, de ellos se desprenden dos posturas. Por un lado, desde el modelo de racionalidad técnica se le confiere a los contenidos todo el peso en cuanto a los saberes obligatorios que debe adquirir la persona estudiante. Estos contenidos se han centrado en la transmisión mecánica de conceptos y hechos desprovistos de estrategias, procedimientos y secuenciación. Desde esta óptica, los contenidos adquieren un carácter nemotécnico y se cuestiona si tienen utilidad para la vida, si se olvidan rápidamente o son permanentes.

Tyler (1973) señala que los contenidos deben permitir el desarrollo de conocimientos que se transformen en habilidades y destrezas para que los y las estudiantes puedan resolver situaciones cotidianas. Deben ser próximos a la experiencia de los sujetos y al contexto social donde se ubica esta. Por su parte, Gimeno (2010) indica que algunos "progresismos" ingenuos han cometido el error de negar el contenido en sí mismo sin comprender su alcance y naturaleza, por lo que el debate no es el de pedagogía de contenidos frente a una pedagogía vacía de ellos, sino una basada en los contenidos propuestos y la metodología con la que estos se enseñan y se aprenden. En suma, los 
contenidos en el diseño curricular deben estar constituidos por tres cualidades: la significatividad, la relevancia y la atracción hacia lo que se espera aprender.

Con respecto a la actividad, Linuesa (2010) señala que las actividades constituyen el elemento más específico y relevante del aula, por ello cuando se plantean, se redibuja, de alguna manera, el diseño de la práctica. Para esta autora, las actividades deben mantener un diálogo continuo con los contenidos, los medios y los recursos disponibles, deben prever adecuación espacial y temporal, secuenciación, equilibrio entre los tipos de actividades y coherencia entre ellas. Las actividades deberán poseer ciertas características, explicadas por Angulo (2005) citadas en Gimeno 2010: ¿Qué deberíamos enseñar? Tópicos generativos: cuestiones, conceptos, temas e ideas que aportan suficiente profundidad, significatividad, conectividad y variedad de perspectivas para apoyar el desarrollo de la comprensión del alumnado. ¿Cómo enseñar para la comprensión? Mediante actuaciones de comprensión: el alumno aplica el conocimiento a situaciones específicas y variadas. ¿Cómo pueden los docentes y el alumnado conocer lo que se ha comprendido? Evaluación continua: esta debe ser un proceso muy distinto a la administración de cualquier tipo de pruebas o exámenes escritos. Debe ser un proceso interactivo entre el docente y el alumnado, en un contexto de aprendizaje que anime a la reflexión sobre el trabajo y la actividad realizada (pp. 340-341).

Por último, la evaluación es el componente curricular más discutido pero también el que más resistencia ofrece al cambio. Algunos componentes curriculares han logrado modificaciones, pero la evaluación parece mantenerse estática. Eisner (1998) señala que no hay consensos, lo que muestra parte de la complejidad que abarca el campo de la evaluación y el peso de la tradición. Lo que preocupa de esta inmovilidad es que si las concepciones de la evaluación siguen la línea tradicional, que evoca el examen como acción puntual y aislada convertida en recurso para medir el aprendizaje, estaríamos obviando la sustancia generadora de oportunidades que puede ofrecer la evaluación, porque "ella misma es recurso valioso de aprendizaje, que asegura el aprendizaje" (Méndez, 2001, citado por Álvarez 2010, p. 356).

Lo importante no solo es contestar ¿qué evaluar? sino cómo y cuándo hacerlo. No se puede sostener el tratamiento de la evaluación como separada de todo el proceso, al contrario, la evaluación extiende sus beneficios cuando se considera en el proceso. De este modo, Tyler (1973) señala que se requiere evaluar porque docente y estudiante necesitan mejorar a partir del conocimiento que esta aporta, necesitan reforzar su aprendizaje con la toma de conciencia sobre su propio progreso y ese papel lo desempeña la evaluación en su 
función formativa. En la medida en que el estudiante es consciente de su aprendizaje y lo internaliza de manera progresiva, será capaz de activar sus conocimientos y rendimiento de forma autónoma.

La clave del cambio para orientar la evaluación radica en dar a los profesores autonomía profesional, oportunidades para poner en práctica sus conocimientos adquiridos, experiencia y quehacer didáctico diario, y contacto con aprendizajes alternativos que generen otras respuestas por parte del estudiantado.

Sin duda, los diseños curriculares son muy diversos y en ellos se plasman unas concepciones particulares según el contexto. Lo que sí debe ser común a todos es la posibilidad de apertura para analizarlos, reflexionarlos y constatar que se establecen vinculaciones sólidas y coherentes, comprender que el currículum es un cuerpo vivo en el que cada componente cumple una función y si alguno falla los demás también. Reafirmar la idea de que el currículum no es solo un documento en papel que instrumentaliza metodológicamente lo que hay que enseñar, sino que de él se desprende una representación de la acción y si ella no es fiel al devenir de su audiencia (en nuestro caso, docentes y estudiantes), si no parte de sus inquietudes, de lo nuevo que surge, de sus vivencias diarias, del tiempo y las dinámicas particulares, estaríamos reproduciendo un germen que desmotiva y desempodera, en lugar de democratizar y hacer avanzar.

\subsection{La enseñanza de la escritura}

¿Qué ha sucedido con la enseñanza de la escritura en la escuela? Cassany (1999) menciona un sinnúmero de tratamientos teóricos, pedagógicos y poco didácticos que se desprenden de trabajos diversos realizados por investigadores acerca de la escritura en la escuela. A continuación, una síntesis:

1. Las prácticas de escritura ponen énfasis en la corrección gramatical y las cuestiones ortográficas, sintácticas y morfológicas.

2. Escribir es una herramienta de evaluación y no un proceso de aprendizaje.

3. La escritura está más relacionada con la forma que con el contenido. Lo que debe enseñarse son las normas y las sutilezas estilísticas, antes que los aspectos de organización y desarrollo de ideas.

4. No se enseña a escribir para pensar y para aprender. La mayor parte de las producciones escritas que genera el aprendiz tienen una función registrativa (toma de apuntes, trabajos) u organizativa (certificativa: exámenes). El alumnado dedica poco 
tiempo a escribir, carece de modelos reales y orientaciones procesuales de lo que debe hacer (cómo debe planificar, cómo debe reflexionar sobre lo que escribe y cómo debe revisar). La práctica reiterada de esas funciones transfiere al estudiantado la idea de que la composición es una tarea mecánica, memorística y poco creativa.

5. Se enseña a escribir en soledad. Se escribe en silencio e incluso proscribe la conversación.

6. Se enseña que la "verdadera" escritura debe ser la analógica, con lápiz y goma de borrar, aunque la forma más frecuente en la actualidad sea la digital. En este sentido, las prácticas compositivas deberían adaptarse a la evolución social y tecnológica, solo de esta manera el alumnado tendrá la sensación de que está usando y aprendiendo un instrumento útil y actual.

7. Ausencia de planificación y revisión. En un contexto real, el autor toma decisiones sobre lo que va a escribir (tipo textual, extensión, destinatario, propósito, tema), pero el modelo tradicional de escritura se limita a la textualización.

8. Heterodirección. Al elegir la consigna de escritura y al gestionar directamente la planificación y la revisión del texto, la personal docente dirige, conscientemente o no, la conducta de la persona estudiante.

9. Sesenta minutos para redactar o construir un escrito es poco tiempo. En la vida real necesitamos mucho más para escribir. Al tener que resolver en tan poco tiempo las tareas de escritura, el aprendiz interioriza que no puede perder tiempo planificando o eligiendo entre varios esquemas posibles, sino que debe terminar su texto en el mismo momento en que lo produce.

10. Ausencia de destinatarios reales. El receptor único de los textos del aprendiz es la persona docente. Aunque las consignas de composición propongan situaciones verosímiles, el contexto académico real se impone al ficticio. La ausencia de lectores reales extraescolares o de lectores intermedios limita el interés por la escritura (Cassany, 1999, pp. 112-136).

\subsubsection{Nuevas tendencias con respecto a la enseñanza de la escritura}

Diversos investigadores en la didáctica de la escritura Cassany (1999); Fontich (2013); Ribas, Milian, Guasch y Camps (2002) señalan que durante décadas las representaciones mentales de la comunidad educativa sobre la escritura difieren, en parte, de las prácticas reales. La concepción del escritor como artista de las letras, que se aísla del mundo para 
crear su obra, la cual se concibe como un producto acabado, con un estilo complejo y una ortografía impecable se contrapone a los estudios que han realizado estos autores en situaciones reales de trabajo de aula, en las cuales "la escritura se relaciona con componentes cognitivos, lingüísticos y sociales que superan con creces las estrechas preocupaciones por la normativa" (Cassany, 1999, p.107).

Para lograr una auténtica enseñanza de la escritura debe partirse de un marco pragmático, sociocultural y cognitivo. El alumnado aprenderá a escribir cuando los espacios de aprendizaje les cedan la palabra, se valoren sus capacidades variadas y se les deje actuar desde cualquier rol que asuman: "ayudar a mi igual", "ayudar al que sabe menos", “dejarme ayudar por el que sabe más”, “movilizar lo que sé” (Van Lier, 2004). En esta línea, la didáctica de la lengua plantea que no puede prescindirse de los contextos reales o verosímiles en que se utiliza la lengua para enseñar la escritura, debe incluirlos en actividades prácticas como elemento esencial del proceso comunicativo. Al mismo tiempo, uno de los propósitos educativos para desarrollar una enseñanza de la escritura pertinente es que su aprendizaje sea facultativo, es decir, si los aprendices comprenden el proceso escritural serán capaces, según Van Dijk (1997), de discernir y de ser críticos ante los discursos ajenos, estarán preparados tanto para interpretar los hechos como para deconstruir las interpretaciones oficiales y elaborar las suyas propias.

Para Fontich $(2010,2013)$, los propósitos de la enseñanza de la escritura desde un enfoque tradicional se sustentan en dos principios que deben cambiar. El primero tiene que ver con la ausencia de una explicación clara entre el paso de una gramática pedagógica o funcional que tiene que desarrollar el aprendiz, como usuario de lengua, con las gramáticas formales de la lingüística, que pretenden elaborar teorías del lenguaje. El segundo, estrechamente relacionado con el primero, se centra en enseñar una gramática desvinculada de los contextos reales de comunicación en los que participa el alumnado.

En este sentido, no se estaría desestimando la enseñanza del sistema gramatical; por el contrario, conocerlo y saber utilizarlo es importante para mejorar el uso, ese conocimiento debe estar en contacto con los contextos reales de comunicación y debe darse lo que estos especialistas llaman conocimiento metalingüístico o reflexión metalingüística, que permite al estudiantado hablar de los fenómenos gramaticales para aprender gramática, no a la inversa, aprender gramática mecánicamente para comprender los fenómenos gramaticales.

En consecuencia, estas reflexiones han movilizado iniciativas y cambios, como la creación de secuencias didácticas o el trabajo por proyectos para abordar la enseñanza de la 
escritura y la lectura. Los investigadores antes mencionados se refieren ampliamente a esta pedagogía por proyectos, la cual se convierte en un "instrumento metodológico que facilita el desarrollo de actividades extensas de escritura" que, con respecto a las breves e intermitentes redacciones habituales, ofrece al estudiantado las siguientes ventajas: motivos para escribir, situaciones de comunicación real, fuerte integración de objetivos globales y concretos, organización cooperativa del trabajo y actividades en las que se dan todas las fases de la escritura (Colomer, Ribas y Utset, 1993, p. 24).

\subsection{La enseñanza de la lectura}

Aliagas, Castellà y Cassany (2009) señalan que

leer y escribir son actividades humanas específicas, situadas histórica, social y personalmente en la vida de cada individuo y comunidad según sus intereses y necesidades. Por lo tanto, existen formas de leer y de ser lector que son socialmente estructurantes, y cada individuo se las apropia en un proceso de enculturación que es particular, íntimo y creativo. (p. 99)

Sin embargo, esta es una noción de lectura actual, surgida de la postura sociocultural para entender la práctica de la lectura y no ha sido ni ha dirigido siempre los procesos de enseñanza de la lectura. En algunas propuestas curriculares, las concepciones más tradicionales siguen asociando la lectura a una tarea individual en la que se desarrollan destrezas cognitivas básicas como anticipar, inferir y formular hipótesis, en pocas palabras, la práctica de la lectura se ha entendido como una alfabetización básica que consiste en leer y comprender.

Ahora bien, La propuesta sociocultural es una corriente teórica que comprende la lectura como una práctica social, en la que el aprendiz comprende un significado, adopta un rol, construye una imagen y participa en una determinada organización de la comunidad. Por ejemplo, se leen y discuten los textos del ámbito más cercano para mostrar el interés y la utilidad social de la lectura; se integra la escritura y la lectura con el resto de códigos, como la imagen, el sonido, los textos multimodales (chats, páginas web, revistas, cómics, redes sociales); se adopta una actitud crítica, en la que comprender requiere construir el contenido pero también descubrir puntos de vista o valores subyacentes del texto (la ideología); además, se fomenta la discusión personal, la elaboración de ideas propias y el diálogo, para que el aprendiz desarrolle sus prácticas de forma autónoma (Cassany, 2009, pp. 18-22). 
La propuesta sociocultural para abordar la enseñanza de la lectura es aún más analizada y profundizada por Colomer (2005); la autora realiza una distinción trascendental entre dos términos que delimitan los espacios o ámbitos de lo literario y lo didáctico de lo literario. Colomer (2005) indica que el cambio de óptica de la forma habitual (de la enseñanza de la literatura a la educación literaria) define visiblemente los caminos. Pero argumenta que el traslado de estos principios a la escuela y su desarrollo coherente ha sufrido múltiples confusiones y problemas; por ejemplo, el conocimiento histórico e hiperformalizado de lo literario sigue vigente en una mezcla de propuestas constructivistas, psicolingüísticas, que no siempre resultan compatibles.

Desde estas líneas de pensamiento, podríamos afirmar que la propuesta sociocultural es integradora y posee unos principios que dialogan no solo con saberes escolares, sino también con saberes externos a la escuela. Desde esta postura, leer es una forma de acción social mediada textualmente y en ella participan dos prácticas: Las prácticas letradas dominantes: engloban las actividades de lectura y escritura que están legitimadas por una institución como una manera apropiada de usar el lenguaje; y las prácticas letradas vernáculas: formas de lectura y escritura que usan un lenguaje no necesariamente estandarizado, aprendidas informalmente e híbridas, por lo que se refiere a las características textuales, y autogeneradas por los individuos (Aliagas et al., 2009, p. 99).

Para concluir este apartado es importante señalar que los aspectos antes esbozados, fueron clave para realizar las tareas de análisis de las áreas de escritura y lectura del currículum de secundaria en Costa Rica, mediante la metodología de redes sistémicas que se detalla en el siguiente apartado sobre el diseño de la investigación.

\section{Diseño de la investigación}

La investigación realizada se enmarca en el ámbito de la Didáctica de la lengua y la literatura. Es un trabajo cualitativo que parte de dos preguntas: ¿Qué contempla el Programa de Estudios de Español de séptimo año, en Costa Rica, para la enseñanza y el aprendizaje de la lectura y la escritura?, ¿Qué cambios conviene introducir en la propuesta curricular de secundaria, que interrelacionen los ámbitos de la lectura y la escritura para lograr una enseñanza del español coherente y significativa?

Para contestar a estas preguntas, el estudio se desarrolló en cuatro fases. En la primera se realizó una exploración y sistematización de información para describir el contexto educativo costarricense y los Programas de Estudio de Español de secundaria. En la 
segunda fase se exploraron y sistematizaron las investigaciones realizadas en el ámbito de la didáctica de la lengua y la literatura en torno a la enseñanza de la escritura y la lectura en secundaria, así como en el ámbito curricular. En la tercera fase se realizó un análisis en profundidad del currículum de Español de séptimo año, contrastándolo con los avances investigativos en el campo de la didáctica de la lengua y la literatura, sintetizados y debatidos en el marco teórico del trabajo. De este contraste (cuarta fase), se establecieron y delimitaron las modificaciones que podrían contemplarse para posibles giros de cambio de la propuesta curricular.

\subsection{Selección de los datos para el análisis}

Los tres criterios principales de selección para analizar el currículum de séptimo año fueron: A) La transición experimentada por los estudiantes al salir de primaria y entrar a la secundaria. Esta transición es un período crítico debido al salto al vacío que experimenta el estudiantado al pasar de una metodología escolar a otra en la que los componentes curriculares y didácticos no dialogan. B) Los altos índices de deserción en séptimo año de la secundaria pública en la última década. Aunque el objeto de estudio de nuestra investigación no fue explorar la multicausalidad de la deserción en la secundaria, varios estudios indican que sí es importante analizar cada factor determinante identificado como posible fuente de deserción y exclusión escolar. En nuestro caso, fue vital la revisión y el análisis del programa de estudios de español, debido a las características y antecedentes de deserción que se presentan en este nivel y, de esta manera, descartar o considerar si el planteamiento curricular podría ser un factor que contribuye a la deserción. C) Debido a la limitación espacio-temporal de esta investigación, fue imposible analizar el currículum oficial de español en su totalidad, por lo tanto, se contempla para el análisis, únicamente la presentación del currículum, su fundamentación, las áreas de escritura y lectura y el corpus de lecturas literarias obligatorias.

\subsection{Métodos de organización y análisis de la información}

Ante una postura de aproximación cualitativa a los datos, se buscó un método que respondiera a la visualización de una especie de fotografía curricular que permitiera observar, describir e interpretar el tratamiento de la escritura y la lectura en el currículum. Esto fue posible a través del método de redes sistémicas de Bliss, Monk y Orgbon (1983). 
Este método facilita un marco de entendimiento que permite sistematizar la información, ver el todo y las partes a través de un lenguaje común y eficaz (ver anexos 1 y 2).

El método de las redes sistémicas deriva de la lingüística sistémica y utiliza los recursos semánticos del lenguaje para establecer un significado detrás de cada palabra escrita que no está directamente expresado. Según sus autores, Bliss et al., (1983) las redes sistémicas que resultan de un análisis muestran la dependencia e independencia entre ideas y se establece un lenguaje gráfico común. Por lo general, se comienza con la lectura detallada de los textos que se pretende analizar y luego se establece un número de categorías por medio de las cuales se podrían organizar los datos ya que se deducen del proceso de lectura. Estas categorías pueden estar previamente definidas por los investigadores de acuerdo con los puntos clave que se pretenden analizar.

En esta línea, el método de redes sistémicas establece el cómo buscar, cómo encontrar, cómo integrar y, además, favorece el análisis y la interpretación.

En el caso de nuestro estudio, el análisis de la información del documento curricular se dividió en dos partes. El proceso de análisis de la primera parte se realizó mediante una descripción analítica de la información. La presentación y la fundamentación del currículum oficial se analizó de forma general, mediante cuatro categorías de análisis: estructura del apartado, enfoque de enseñanza presente-subyacente, fines y objetivos de la escritura y la lectura y concepción didáctica de ambas prácticas. Posteriormente, se realizaron unas conclusiones de lo encontrado en las áreas descritas; esto con el fin de contrastar lo que plantea el currículum desde el discurso oficial y los hallazgos en el análisis de las áreas de escritura y lectura.

Para la segunda parte se confeccionaron tablas de análisis previas a la construcción de las redes sistémicas (ver anexos 1 y 2). Estas tablas permitieron organizar y sintetizar los datos correspondientes a los componentes curriculares presentes en el currículum oficial. El momento de organizar y analizar la información en las áreas de escritura y lectura del currículum oficial de séptimo año, surgieron ocho redes sistémicas generales. Cuatro redes corresponden a los cuatro objetivos de escritura y las otras cuatro redes, a los cuatro objetivos de lectura del plan de estudios. En cada red se analizaron los cinco componentes curriculares: objetivos, contenidos, actividades de aprendizaje, valores y actitudes y evaluación. A su vez, estos cinco componentes curriculares se analizaron a la luz de las cuatro categorías elegidas para la confección de las redes sistémicas: tipo de (objetivo, contenido...), meta, énfasis y ausencia. El proceso de construcción requirió de un total de 
más de cuarenta redes (cinco para cada componente curricular por cuatro objetivos de lectura y cuatro de escritura) y ninguna quedó finalizada en el primer intento, fue necesario un proceso recursivo en el que cada mirada analítica realimentara la red, hasta recoger todos los datos posibles para emprender el análisis.

A continuación, en la figura 1, se ofrece un ejemplo de una red sistémica correspondiente al primer objetivo de lectura.

Figura 1. Esquema de red sistémica del objetivo del área de lectura del Programa de estudios de español de Costa Rica, 2009

\begin{tabular}{|c|c|c|c|c|c|c|}
\hline \multirow{7}{*}{$\begin{array}{l}\text { Objetivo } 1 \\
\text { Área: Lectura }\end{array}$} & \multirow{7}{*}{$\begin{array}{l}\text { Aplicar el análisis } \\
\text { literario como una } \\
\text { forma de explorar los } \\
\text { múltiples sentidos del } \\
\text { texto. }\end{array}$} & \multirow{7}{*}{ Tipo de objetivo } & \multicolumn{4}{|c|}{$\begin{array}{l}\text {-del dominio cognitivo } \\
\text {-objetivo de aplicación. Para que el estudiante pueda aplicar } \\
\text { antes tuvo que aprender una información y luego aplicarla } \\
\text { a una situación nueva. } \\
\text {-objetivo de acción pero de connotación mecanicista. } \\
\text { Primero se aprenden las categorias de análisis literario, luego se aplican al texto literario. } \\
\text { de Bloom }\end{array}$} \\
\hline & & & \multirow{6}{*}{$\begin{array}{c}\text { Aplicar una } \\
\text { estructura de } \\
\text { análisis } \\
\text { literario para } \\
\text { acercarse al } \\
\text { texto literario }\end{array}$} & - Énfasis & $\begin{array}{l}\text { Modelo } \\
\text { estructuralista }\end{array}$ & $\begin{array}{l}\text {-Se centra en el texto. } \\
\text {-Aplicación mecánica de } \\
\text { un procedimiento de } \\
\text { análisis literario (afán de } \\
\text { categorización). }\end{array}$ \\
\hline & & & & & {$\left[\begin{array}{l}\text { Corrientes: } \\
\text { lingüística y } \\
\text { psicolingüistica }\end{array}\right.$} & $\begin{array}{l}\text {-Aprender el código } \\
\text {-Ampliar el vocabulario } \\
\text {-Anticipar } \\
\text {-Inferir los significados no literales } \\
\text {-Formular hipótesis } \\
\text {-Lectura como técnica individual }\end{array}$ \\
\hline & & & & & $\begin{array}{c}\text { Enseñanza de la } \\
\text { literatura }\end{array}$ & Conocimiento histórico e hiperformalizado de lo literario \\
\hline & & & & & $\begin{array}{l}\text { Corriente } \\
\text { sociocultural }\end{array}$ & {$\left[\begin{array}{l}\text {-Literatura como práctica social: el aprendiz comprende } \\
\text { un significado, adopta un rol, construye una imagen y } \\
\text { participa en la comunidad. }\end{array}\right.$} \\
\hline & & & & Ausencia & $\begin{array}{l}\text { Educación } \\
\text { literaria }\end{array}$ & $\begin{array}{l}\text { - Contempla un mapa literario en el que intervienen: El } \\
\text { lector como centro, el texto y el contexto. } \\
\text {-Se interesa por el bagaje literario que traen los alumnos. } \\
\text {-Experiencia en la que participa un texto especifico y un } \\
\text { lector particular. }\end{array}$ \\
\hline & & & & & $\begin{array}{l}\text { Relaciones con el } \\
\text { contexto }\end{array}$ & {$\left[\begin{array}{l}\text { El análisis estructural de los textos literarios se } \\
\text { convierte en una disección arbitraria que } \\
\text { impide las relaciones con la realidad y } \\
\text { contextos próximos de los estudiantes. }\end{array}\right.$} \\
\hline
\end{tabular}

Fuente: Elaboración propia (2014). Red sistémica. Anexo 2, p. 30

Una vez finalizado el proceso de la construcción de las redes, se procedió a imprimirlas y ubicarlas en un pizarrón grande, donde se realizaron lecturas verticales, horizontales, específicas y globales, todo con el fin de realizar el análisis mediante líneas convergentes, relaciones, recurrencias y los vínculos o disociaciones entre el discurso del texto curricular y el discurso didáctico empleado para enseñar lengua y literatura. 


\section{Resultados}

En el análisis de contenido realizado en las áreas de escritura y lectura, los cuatro objetivos de escritura y los cuatro objetivos de lectura presentan similitudes y concurrencia de características.

1. Todos los objetivos, tanto los de lectura como los de escritura, son del dominio cognitivo, de síntesis, aplicación y conocimiento, según la clasificación taxonómica de Bloom (1973).

2. Los objetivos redactados mediante verbos observables u operativos como: producir, aplicar, distinguir, desarrollar y propiciar, en apariencia son verbos de acción pero tienen una connotación mecanicista. Pertenecen a los conocimientos que se adquieren mediante los niveles de memorización, comprensión y aplicación pero no avanzan hacia los niveles de análisis, evaluación y creación, en los que se considera se alcanza el aprendizaje significativo.

3. Los objetivos de escritura y de lectura no poseen un orden lógico secuencial.

4. Todos los objetivos hacen explícita una tendencia normativista. Además, los cuatro objetivos de escritura son uno mismo, todos se abocan a la producción de textos escritos desde una concepción tradicional. No se presenta un objetivo distinto, con otras metas que permitan abordar otras áreas del campo, como elaborar planes de acción para escribir, imaginar o contactar destinatarios reales, valorar las producciones en un contexto comunicativo determinado, organizar cooperativamente el trabajo de escritura, desarrollar una puesta en común de ideas, evaluar las producciones, compartirlas, entre otras.

5. Los objetivos de escritura enfatizan en la escritura del texto como producto. Se plantea la tarea de producción textual, pero sin considerar etapas previas de escritura como: recopilación de información, organización de ideas, escritura de borradores, práctica de la recursividad como táctica para revisar, mejoramiento y reformulación del texto, autorevisión y coevaluación como procedimientos para aprender y avanzar en la adquisición y el desarrollo de saberes y destrezas propias de la escritura.

6. También se enfatiza en la norma rígida y en la gramática formal para escribir textos, así como en la escritura más relacionada con la forma y la aplicación de reglas morfosintácticas que con el contenido o construcción del significado. 
7. En los objetivos existen ausencias en cuanto a concebir el texto escrito como proceso. No se percibe la norma flexible, ni la reflexión acerca de la lengua durante el proceso de escritura; la gramática es formal, no funcional.

8. Todos los objetivos se orientan al modelo estructuralista de la literatura y a las corrientes lingüísticas y psicolingüísticas. Se centran en el texto y en una aplicación mecánica de un procedimiento de análisis. La enseñanza de la literatura se centra en su conocimiento histórico e hiperformalizado de lo literario.

9. Los objetivos de lectura se confunden con los de literatura. Carecen de una corriente sociocultural que oriente la enseñanza.

Estos hallazgos en el análisis de los objetivos muestran concepciones poco contextualizadas en cuanto a las funciones vitales que debe ejercer el componente curricular de los objetivos, como agente que ordena el curso de la acción, como componente que guía la acción docente y que constituye el marco de referencia para desarrollar de forma organizada y coherente el proceso de enseñanza. La explicitación de lo que se va a realizar desde la perspectiva del aprendiz y su contexto es tarea del objetivo y esta parece estar ausente en los resultados del análisis realizado.

Aunque en los objetivos que se mencionan en el apartado de la fundamentación del documento curricular se muestra una corriente constructivista que ordena la consecución de saberes, los ocho objetivos referidos a la lectura y la escritura planteados en la parte operativa del currículum, poseen una naturaleza conductista y mecanicista que pone en duda la racionalidad y cientificidad del proceso de enseñanza. El hecho de hallar esta contrariedad de enfoques en los objetivos —constructivismo/conductismo-, supone un primer debilitamiento en cuanto a la función que deben ejercer el currículum y, además, nos hace preguntarnos si existe una confusión entre fines, conductas y objetivos que permea 0 condiciona todos los demás componentes curriculares en un "efecto dominó".

La enseñanza por objetivos o la pedagogía por objetivos (Tyler, 1973; Gagné, 1979; Bloom, 1973), es un modelo ya cuestionado por diversos teóricos (Ammons, 1964; Gimeno, 2010; Stenhouse, 1987;) debido a esa tendencia conductista y eficientista. Esta tendencia se visualiza claramente en el planteamiento de los objetivos de enseñanza de la escritura y la lectura del documento curricular de español, ya que se espera que el estudiantado alcance de manera mecánica saberes formales de escritura y de lectura, los aprenda, los practique y los repita cuando la persona docente lo solicite. En este sentido, las tareas o metas 
encontradas en los objetivos como producir, aplicar, distinguir y desarrollar no son suficientes para alcanzar progresiones en la adquisición de una competencia como escritores o lectores; se requiere del engranaje de otros múltiples saberes que van más allá de tareas como memorizar, comprender y aplicar. Es necesario que los estudiantes vivan experiencias que les permitan resolver, juzgar, seleccionar, criticar, optimizar e interpretar; para ello, los objetivos deben plantearse de forma secuencial y procesual.

Los objetivos planteados son conceptuales, se concibe al estudiante como productor de textos escritos antes de enfrentarse a etapas previas de escritura; no se concibe como constructor o creador de textos. Este aspecto podría obstaculizar el incremento de las capacidades compositivas y creadoras de la persona estudiante, pues se hace visible la carencia de modelos reales y orientaciones procesuales en cuanto a cómo planificar un escrito, cómo reflexionar sobre él, cómo valorarlo, revisarlo y compartirlo.

Con respecto a los objetivos de lectura de textos no literarios, se considera aquí el momento para desarrollar la comprensión lectora y la aplicación de todos los niveles de lectura, como si la lectura de textos literarios no participara también de esta dinámica de comprensión e interpretación. La comprensión lectora es un objetivo siempre transversal en todas las áreas curriculares y debe practicarse en todo momento.

Sin embargo, en los objetivos planteados, parece darse un distanciamiento entre el tratamiento de la comprensión lectora de los textos no literarios, que consiste en: aprender el código, ampliar el vocabulario, anticipar, inferir los significados no literales, formular hipótesis y realizar el ejercicio de lectura como técnica individual; mientras que para los textos literarios se debe centrar en otro tratamiento, como lo es la aplicación mecánica de un procedimiento de análisis literario. Estos aspectos muestran una ausencia de lo que debe ser una educación literaria centrada en el lector. Aún se concibe la enseñanza de la literatura como un conocimiento histórico e hiperformalizado en el que se reconocen y memorizan características de géneros y movimientos literarios, y no en una enseñanza que permita aprender a leer literatura.

De esta manera, el análisis literario se convierte en un ejercicio de categorización en el que no se concibe la literatura como una práctica social en la que el aprendiz comprende un significado, adopta un rol, construye una imagen y participa en la comunidad. Tampoco se contempla un mapa literario en el que intervienen: el lector como centro, el texto y el contexto, ni se muestra interés por el bagaje literario que trae el estudiantado. 
Con respecto a los contenidos, el documento curricular asume al componente desde su carácter nemotécnico, como un listado de información y saberes que debe adquirir la persona estudiante. Los contenidos, todos son conceptuales y se presentan desarticulados, sin orientaciones claras y significativas para el aprendiz. Es posible que su puesta en práctica repita el patrón del currículum intentado, que promueve la memorización de conceptos, la instrumentalización del saber y la reproducción de una metodología vacía y estática, incapaz de integrar los significados previos del estudiantado, de ilusionarlo, de problematizar y de despertar inquietudes ante los conocimientos que se le ofrecen.

Durante todo el análisis de los componentes curriculares, existe una tendencia marcada y reiterada de copiar de forma literal la información de un componente curricular a otro. Por ejemplo, los contenidos del objetivo 1 (de escritura) son una copia literal de los procedimientos y los contenidos del objetivo 2 (de lectura) son una copia literal del objetivo 2 . Esta situación genera poca credibilidad por cuanto no se logra una concreción de cada componente, y no solo se tiende a confundir, sino a sustituir un elemento por otro de forma indiscriminada.

Lo mismo sucede con los contenidos de lectura. Aunque se menciona en la fundamentación del currículum que estos se abordarán desde una noción crítica, creadora y dinámica, estas nociones no se reflejan en los contenidos propuestos, no se pone énfasis en enseñar a pensar, a que el lector se enfrente a la aventura de descubrir significaciones o sentidos múltiples, de enriquecer y recrear el texto, de aplicar experiencias e imaginación a partir del texto y de expresar sus propios significados. Al contrario, en los actos de leer y escribir, se promueven tareas mecánicas, poco significativas, que consideran al texto como un todo hecho, intocable y venerable, como un objeto que hay que sobrellevar. En esta línea, los contenidos de escritura y lectura analizados en la parte operativa del currículum y las nociones o componentes que dicen considerarse para abordarlos en el apartado de fundamentación se oponen de manera drástica.

Por otra parte, de todos los componentes curriculares analizados, los procedimientos son los más estáticos pues se repiten casi de forma idéntica de un objetivo a otro. Este aspecto sugiere algunos datos que permiten explicar el porqué de esta condición que se analiza después de mostrar los resultados en las ocho redes de procedimientos:

1. Todos los procedimientos que se presentan son conductuales (realizar tareas para la adquisición de conductas), excepto el del objetivo 3 de lectura, que es procedimental 
pues permite la ejecución de tareas como la interpretación, la emisión de juicios críticos y la producción y recreación de textos.

2. Aunque se menciona que los procedimientos son conductuales, se da un fenómeno que también permite afirmar que no se presenta un procedimiento coherente de acciones ordenadas y orientadas a la consecución de procesos. El fenómeno del caso ocurre porque los procedimientos son el mismo listado de contenidos propuestos en el componente curricular de contenidos. No se especifica si se trata de un procedimiento simple, complejo, de pocos o muchos pasos; de hecho los pasos no son identificables.

3. Lo que el currículum asume como procedimientos de escritura y de lectura se centra en tareas mecánicas, las mismas que se plantean en los contenidos: clasificar, aplicar, escribir, localizar, ordenar, repasar, deducir, corregir, elaborar, resumir, producir, ejercitar. En todas las redes sistémicas de los procedimientos, lo único que cambia son los verbos para asignar las tareas por realizar, excepto en el objetivo 3 de lectura, en el que se proponen tareas más participativas y se presentan principios metodológicos, tales como la socialización, la actividad, la participación, la creatividad, la optimización y la integración. En este objetivo, el énfasis se da en la experiencia con la literatura. En los otros tres objetivos, se centra en el saber literario, no en la experiencia con la literatura.

4. El componente curricular de procedimientos pone énfasis en la forma del texto no en la construcción del significado del texto.

5. En todas las redes de procedimientos existe una ausencia en cuanto a principios y estrategias didácticas específicas posibles: Agrupación: ¿Cómo se espera colocar al estudiantado en diversas trayectorias de aprendizaje? ¿Se espera que aprenda de forma individual, en pequeños grupos o con toda la clase?, Ubicación: ¿Se espera que el estudiantado aprenda en clase, en la biblioteca, en casa o en otro lugar?, Tiempo: ¿Cuánto tiempo se espera invertir en las tareas específicas de aprendizaje?, Materiales y recursos: ¿Con qué se espera que aprenda el estudiantado?

6. La interacción que se propone es unidireccional: docente-activo, estudiante-pasivo. El rol de la persona docente consiste en dar contenidos. El rol de la persona estudiante consiste en recibir contenidos (excepto en el objetivo 3 de lectura).

$\mathrm{Si}$ se observan las redes de contenidos y se comparan con las redes de procedimientos, se revela otro aspecto que se trae abajo la suposición de que en el 
currículum se maneja una idea de procedimientos como pautas generales de actuación. Este hecho se observa al corroborar que los contenidos y los procedimientos son los mismos; es decir, el componente curricular de procedimientos se vuelve estéril porque repite el listado de contenidos. Esto sugiere que no existe procedimiento como tal, este se reduce a impartir contenidos desprovistos de procedimientos.

Esta dinámica plantea que la propuesta curricular no solo carece de procedimientos articulados que orienten la práctica de enseñanza, sino también de un marco para elegir las estrategias y, con ello, las actuaciones del alumnado. En este sentido, no se respalda la labor de programación didáctica del profesorado, no se le ofrecen posibilidades o sugerencias para ampliar su ejercicio didáctico. Lo que sugiere el currículum intentado es impartir y reproducir de manera mecánica los contenidos. Corroborar si los docentes reproducen esta postura en el aula es una futura tarea de investigación, pero este hallazgo en el currículum intentado sugiere que el planteamiento actual para la enseñanza del español, desde su base teórica y organizativa, podría estar debilitando los procesos de una enseñanza articulada y coherente, y el aprendizaje genuino por parte del estudiantado.

También se observa que todos los componentes curriculares tienden a estar en función únicamente de los contenidos. Parece que este componente es el dominante, por lo tanto, la representación de la acción, planteada en el documento curricular oficial, tiende a reducirse a la tarea de impartir contenidos que aseguran el cumplimiento de los objetivos, sin importar el procedimiento, y constatar que se adquirieron a través de la evaluación.

Con respecto a los valores y actitudes hacia la escritura y lectura, las redes sistémicas muestran que estos se reducen solo a aspiraciones, o bien, se confían al profesionalismo de los docentes en el momento de ejercer su práctica. Esta afirmación se confirma al observar que los valores y las actitudes que se intentan promover no se sustentan porque no descansan en procedimientos o procesos que estimulen ambientes de enseñanza óptimos. Los procedimientos no favorecen ninguno de los valores que se esperan del estudiante. Todos los valores y actitudes propuestos en el documento curricular para cada objetivo (con la sola excepción del objetivo 3 de lectura) buscan que el estudiantado adquiera y desarrolle interés, aprecio y disposición por la escritura y la lectura, además del ejercicio de la capacidad crítica y analítica. Sin embargo, la inexistencia de procedimientos priva el desarrollo de ambientes de aprendizaje que permitan adquirir y desarrollar esos valores. A esta situación, se suma la confusión conceptual existente en cuanto a lo que es un valor, una actitud, un procedimiento y un contenido. Pretender que un estudiante sienta aprecio por un 
diccionario, que valore la ortografía o desarrolle conciencia sobre la morfosintaxis sin la oportunidad de experimentar espacios de reflexión relativos a la lengua, enlazada a otros saberes, es una visión reduccionista de entender el desarrollo de valores y actitudes durante la enseñanza. Existe un desconocimiento en cuanto a las concepciones de que los valores y actitudes se adquieren en el proceso si se presentan procedimientos oportunos y contextualizados a la realidad estudiantil.

Finalmente, en las redes sistémicas que analizan el componente de la evaluación, se visualiza que su planteamiento es el más crítico de todos los componentes curriculares. Se encontró que, en la evaluación, se repiten de forma literal los objetivos de enseñanza, esto genera una ausencia contundente con respecto a la forma en la que no se logra concretar este componente en el documento curricular. De igual forma, la evaluación que parece predominar es la evaluación final-sumativa, en la que se corrige la versión final del texto escrito, en el caso de la escritura y se corrobora que el estudiante aplique correctamente la estructura analítica a los textos literarios, en el caso de la lectura.

Esta forma reduccionista de entender ambos procesos no promueve la complejidad del discurso escrito y literario. En ningún objetivo se sugiere la evaluación continua, formativa u otras formas de evaluación. Este delicado hallazgo, que contempla la indefinición del componente, o bien, que sigue una línea tradicional evocadora del examen como acción puntual y aislada para medir el aprendizaje, implica de forma insalvable que se entienda y valore la evaluación como "la sustancia generadora de oportunidades para aprender, que lleva al alumno a participar como actor en la apropiación del saber y a que no permanezca como espectador del mismo" (Álvarez, 2010, p. 359).

\section{Conclusiones}

¿Qué contempla el Programa de estudios de Español de séptimo año, en Costa Rica, para la enseñanza y el aprendizaje de la escritura y la lectura?

- El currículum refleja inconsistencias y confusiones conceptuales o desconocimiento en materia curricular en el planteamiento y organización de objetivos, contenidos, procedimientos, valores y evaluación. Esto se ve reflejado en la repetición o copia literal de los objetivos en el componente de la evaluación, o bien, en las consideraciones erradas de lo que es un valor o actitud, un procedimiento y un contenido de enseñanza. El análisis reveló que existe una carencia contundente en cuanto a la comprensión de la evaluación para los aprendizajes. Las redes sistémicas 
permiten ver que es el componente más crítico del currículum, porque no se hacen explícitas las formas para abordarla o, aún peor, se asume como objetivo de enseñanza lo que es una evaluación.

- Una lectura superficial del documento curricular hace pensar que cuenta con todos los requerimientos: presentación, fundamentación, transversalidad, enfoque general de enseñanza, objetivos, orientaciones para el docente, sugerencias para la evaluación, delimitación de áreas: escucha, expresión oral, lectura, expresión escrita y corpus de lecturas; no obstante, una lectura minuciosa hace constar que, efectivamente, las partes están presentes y todas ofrecen una explicación detallada, pero la gran ausencia tiene que ver con la coherencia entre las partes, sobre todo las incongruencias entre lo que plantea el preámbulo de la fundamentación del documento curricular y lo encontrado en los componentes curriculares, que es de donde deben partir los docentes para elaborar sus programaciones didácticas. Los vacíos conceptuales, teóricos, didácticos y curriculares son la constante, así como la escasa explicitación y poco conocimiento acerca de cómo plantear los contenidos de enseñanza.

- Las corrientes teóricas y los enfoques que sustentan la enseñanza de la escritura y la lectura en el documento curricular analizado presentan una dualidad. En la fundamentación se indica que el enfoque de enseñanza es el socioconstructivismo, y las corrientes teóricas para la enseñanza de la escritura y la lectura contemplan los componentes motivacional, conceptual, lingüístico, pragmático, discursivo, semántico, morfológico y sintáctico, notacional y locutivo. También afirma que promueve lecturas y escrituras críticas, creadoras y dinámicas; que se apela por un estudiante que valore la lengua como factor de desarrollo, que sea activo, participativo, crítico, capaz de intervenir en la toma de decisiones, así como por un docente creador de la necesidad y el placer por leer, escribir, escuchar y hablar, creador de proyectos, polemista, que promueva la imaginación y el juicio crítico.

Sin embargo, en la puesta en común de los componentes curriculares, solo se acogen dos componentes, el conceptual y el morfosintáctico, así como la lectura mecánica de textos no literarios y la lectura estructuralista de textos literarios, dejando desmantelada así la enseñanza de la lengua y la literatura de estudios actuales que expliquen su enseñanza y aprendizaje desde otras visiones más actualizadas, que dejen atrás las nociones 
tradicionales que la han precedido durante décadas. Por otra parte, la persona estudiante es presentada como un ser pasivo que recibe instrucciones, conocimientos desarticulados y descontextualizados, la interiorización de procedimientos muy estructurados para que pueda replicarlos de forma mecánica en otros momentos o bien en las situaciones o tareas estáticas que sugiere el currículum. Además, en la globalidad de los componentes curriculares, se reduce la labor del docente a la de un simple programador de aprendizajes que organiza los contenidos, los imparte y los evalúa. Desde esta situación, la propuesta curricular encubierta, que enseña la estructura de conocimientos y procedimientos, propia del enfoque conductista de enseñanza, es la que impera en el documento curricular y desde esta debilidad se originan contradicciones.

- Las concepciones de enseñanza de la escritura y de la lectura en la fundamentación dicen concebirse a partir del desarrollo de las potencialidades de pensamiento y la competencia comunicativa, pero en los componentes curriculares emergen otras concepciones muy distintas a las planteadas en este apartado. La enseñanza de la escritura y la lectura aún se concibe en marcos de enseñanza tradicionales, centrados en la persona docente, cuya función es prescribir tareas y evaluar el producto final, ejercer como única audiencia, en el caso de la producción de textos escritos y lectura de textos literarios, por parte del estudiantado. La enseñanza literaria se centra en conocer autores, las características de géneros y movimientos literarios, en aplicar estructuras de análisis a los textos en lugar de procurar que quienes estudian aprendan a leer, a apreciar, interpretar, participar, argumentar y ser críticos con su entorno. E texto literario sigue siendo el centro como entidad cerrada y sujeta a estudios formales, aún no se concibe el desplazamiento del texto del centro y al lector en su lugar. La enseñanza de la escritura concibe al escritor como productor de textos, que aplica reglas ortográficas y gramaticales y que espera la corrección del docente. En este sentido, la escritura se entiende desde una perspectiva instrumental y la lectura como un proceso complejo para el que no hay formas definidas para abordarlo. De esta manera, ambas concepciones de enseñanza de la escritura y de la lectura se representan lejos de las intenciones socioculturales y cognitivas que considera la didáctica de la lengua y la literatura. 
¿Qué cambios conviene introducir en la propuesta curricular de secundaria que interrelacione los ámbitos de la escritura y la lectura para lograr una enseñanza del Español coherente y significativa?

Los cambios que conviene introducir en la propuesta curricular de $7^{\circ}$ año de secundaria en Costa Rica consisten en tres grandes giros hacia: una nueva forma de entender el currículum, nuevas formas de enseñanza de la escritura y de la lectura, y una simbiosis entre el discurso didáctico, el discurso cultural y el discurso curricular.

Comprender los alcances y las funciones de una propuesta curricular articulada, conocedora del objeto de enseñanza, al servicio del profesorado en la que este pueda intervenir, crear, modificar y aportar en el ejercicio de su labor, representa una nueva forma de concebir el currículum. El currículum hoy debe transformarse —utilizando la metáfora de Akker, Fasoglio y Mulder (2010) - en una enorme tela de araña inacabada. Esta metáfora concibe el currículum como una estructura viva e intrincada, que se construye formando círculos en los que se conectan intenciones, metas, conocimientos y actividades como un todo coherente. En ese todo, el docente puede ejercer un currículum congruente, tomando y analizando el conocimiento teórico disponible y utilizando el suyo, obtenido de su reflexión sobre su práctica.

Esta integración de saberes podría garantizar programaciones didácticas contextualizadas y pertinentes que favorezcan aprendizajes significativos para el estudiantado. Es evidente que si se desea alcanzar una reconversión metodológica, puesta en marcha por el profesorado, se debe iniciar por la reflexión y la construcción de un marco de actuación curricular en donde se contemplen las innovaciones teóricas y las experiencias prácticas consolidadas como pilares de una enseñanza didáctica de la escritura y de la lectura.

Replantear el currículum costarricense desde los aportes de los avances investigativos de la didáctica de la lengua y la literatura sería una oportunidad para transformar la enseñanza de la escritura y de la lectura. Reconceptualizar el objeto de enseñanza supone reflexiones e intervenciones de docentes, estudiantes, especialistas en el tema curricular y de la especialidad de Español, en el sentido de qué conviene aprovechar esos aportes ofrecidos por las últimas investigaciones en el campo, qué es necesario modificar, de qué manera y cómo se esperan alcanzar esos giros, de forma paulatina pero contundente, con una convicción de que no son cambios superficiales ni parciales, sino sustanciales y que su naturaleza es lenta y escalonada pero progresiva. 
Unido a este aspecto antes descrito, las propuestas curriculares de primaria y secundaria deben establecer puentes de aprendizaje con respecto a la adquisición del metalenguaje por parte de los aprendices. Muchas veces los currículos de primaria se abocan únicamente a la lectura de textos literarios y se delega a la secundaria el aprendizaje de la literatura. Esta condición obstaculiza lo que Grossmann y Tauveron (1999) Ilaman afianzar una lectura interpretativa desde la escuela.

Finalmente, la simbiosis entre los discursos didáctico, cultural y curricular es indispensable para que las personas estudiantes adquieran una competencia comunicativa idónea que les permita enfrentar los retos comunicativos del siglo XXI. Por ello, el currículum debe valorar la escritura y la lectura como prácticas socioculturales; es decir, debe conocer las prácticas que se hacen con la lectura y la escritura fuera de la escuela y establecer conexiones fuertes para que el estudiantado pueda concebirlas como prácticas cotidianas de las que participa siempre. El nuevo orden social regido por la tecnología ha provocado cambios en las prácticas de escritura y lectura y estas no pueden ser obviadas por las propuestas curriculares; por el contrario, es la oportunidad para enseñar a las personas estudiantes a cuestionarse sobre los textos que circulan, quién los construye, desde dónde y por qué. Es una oportunidad para escribir, intervenir, problematizar, leer entre líneas y aprender a interpretar discursos de forma que puedan afianzar su pensamiento y su intervención social.

Consideramos que delimitar y poner en práctica una metodología de investigación como la de redes sistémicas, nos permitió establecer pautas de lectura y análisis de los datos cualitativos que se desprendieron de la propuesta curricular. Esta aportación de índole metodológica, podría permitir a otros investigadores interesados en el análisis curricular, replicarla pues permite lecturas horizontales, verticales, específicas y globales. Asimismo, otra de las aportaciones de la investigación consiste en desmitificar el papel que juega el currículum en el ámbito educativo, afianzar la idea de que no es un simple documento escrito, sino un texto intentado que está vivo porque representa la acción y es trascendental, porque si no organiza los saberes de forma coherente y con profundo conocimiento del objeto de enseñanza provoca una cadena de desaciertos.

En relación con esta aportación, surge una delimitación espacio-temporal del estudio, la cual consistió en no poder explorar otra fase de investigación, que en un primer momento consideramos pertinente, la cual consistía en acercarnos a las lecturas e interpretaciones que hacían los docentes del texto curricular intentado - es decir, el documento curricular 
oficial- y contrastar estos datos con la puesta en práctica de las personas docentes en el aula. Sin embargo, valoramos la posibilidad de contemplar en una primera etapa de investigación, únicamente el análisis necesario en profundidad del documento curricular y, posteriormente, ya con el bagaje y el conocimiento sustancial del currículum aportado durante esta etapa, emprender una segunda etapa de investigación.

De esta manera, la ruta parece abierta a futuras investigaciones que exploren esta temática y, por qué no, a un posible procedimiento para diseñar una propuesta curricular que articule los saberes de la escritura y de la lectura en secundaria. Lo que sí se logró con este estudio fue tener más claro el panorama curricular costarricense y establecer la importancia de construcción de un marco que relacione los saberes de la didáctica de la lengua y la literatura y los saberes curriculares como primer paso para el desarrollo de una educación de la lengua y de una educación literaria meditadas y posibles de ejercer.

\section{Referencias}

Akker, Jan van den, Fasoglio, Daniela y Mulder, Hetty. (2010). A curriculum perspective on plurilingual education. Netherlands: Netherlands institute for curriculum development; Council of Europe.

Aliagas, Cristina, Castellà, Josep y Cassany, Daniel. (2009). “Aunque lea poco, yo sé que soy listo". Estudio de caso sobre un adolescente que no lee literatura. Ocnos, (5), 97112.

Álvarez, Juan Manuel. (2010). El currículum como marco de referencia para la evaluación educativa. En José Gimeno (Comp.), Saberes e incertidumbres sobre el currículum (pp. 355-372). Madrid: Morata.

Ammons, Margaret. (1964). An empirical study of process and product in curriculum development. The Journal of Educational Research, 57(9), 451-457.

Arias, Grettel, Ruíz, Yerlin y Vargas, Lorriane. (2013). La mediación pedagógica para la enseñanza de la literatura en los colegios José Joaquín Vargas Calvo, Elías Leiva Quirós y Miravalle (Memoria de Graduación para optar por el grado de Licenciatura en la Enseñanza del Castellano y la Literatura). Universidad de Costa Rica. San José, Costa Rica.

Barnett, Ronald. (2001). Los límites de la competencia. El conocimiento, la educación superior y la sociedad. Barcelona: Gedisa.

Bliss, Joan, Monk, Martin y Ogborn, Jon. (1983). Qualitative data analysis for educational research: a guide to uses of systemic networks. London: Croom Helm.

Bloom, Benjamín. (1973). Taxonomía de objetivos de la educación. Alcoy: Marfil. 
Calarusso, Guiseppe. (2014). Improbabilità. Recuperado de http://www.giuseppecolarusso.it/improbabilita .html

Casaca, Laura. (2009). Salirse del cascarón: Nuevas tendencias en la enseñanza de la escritura. Revista Kañina, 33(2), 55-70.

Cassany, Daniel. (1999). Construir la escritura. Madrid: Espasa.

Cassany, Daniel. (2009). Para ser letrados. Voces y miradas sobre la lectura. Barcelona, España: Paidós.

Colomer, Teresa. (2005). Andar entre libros. La lectura literaria en la escuela. México, D.F.: Fondo de Cultura Económica.

Colomer, Teresa, Ribas, Teresa y Utset, Marta. (1993). La escritura por proyectos: Tú eres el autor. Aula. La innovación en la enseñanza de la lengua, (14), 23-28.

Eisner, Elliot. (1998). El ojo ilustrado. Indagación cualitativa y mejora de la práctica educativa. Barcelona: Paidós.

Fontich, Xavier. (2010). La construcció del saber metalingüístic: estudi sobre l' aprenentatge de la gramàtica d' escolars de secundària en el marc d' una seqüencia didàctica (Tesis doctoral). Universidad Autónoma de Barcelona, Barcelona, España.

Fontich, Xavier. (2013). La gramática de la primera lengua en la escuela: Reflexiones sobre su enseñanza-aprendizaje y sobre el contenido gramatical escolar. Bellaterra Journal of Teaching \& Learning Language \& Literature, 6(3) 1-19)

Gagné, Robert. (1979). Las condiciones del aprendizaje. México: Interamericana.

Gallardo, Isabel. (2006). La lectura de textos literarios en el colegio. ¿Por qué no leen los estudiantes? Revista Educación, 30(1), 157-172.

Gimeno, José. (1982). La pedagogía por objetivos: Obsesión por la eficiencia. Madrid: Morata.

Gimeno, José. (2010). Saberes e incertidumbres sobre currículum. Madrid: Ediciones Morata.

Gimeno, José. (2010). El currículum en la acción: Los resultados como legitimación del currículum. En José Gimeno, (Comp.), Saberes e incertidumbres sobre currículum (pp. 311-332). Madrid: Ediciones Morata.

Grossman, Francis y Tauveron, Catherine (Eds.) (1999). Comprendre et interpréter les textes á l'école. La lecture á la jonction du cognitif et du culturel (Repères, Número 19). Paris: INRP.

Linuesa, María Clemente. (2010). Diseñar el currículum. Prever y representar la acción. En José Gimeno (Comp.), Saberes e incertidumbres sobre el currículum (pp. 269-293). Madrid: Morata. 
Méndez, Silvia. (2000). La mediación docente en la comprensión de lectura de textos literarios. Revista Educación, 24(1), 89-105.

Murillo, Marielos. (2009). Repensar la enseñanza de la lengua española en la educación costarricense. Los alumnos en el proceso de transición sexto grado/sétimo año. Revista Kañina, 33(2), 133-147.

Ovares, Flora, Alfaro, Jorge, Rojas, Margarita y Mora, Sonia Marta. (1986). La palabra al margen: La enseñanza del español en crisis. San José: Editorial Nueva Década.

Ramírez, Catalina. (2006). Estrategias metodológicas usadas por los docentes de sétimo año en la enseñanza del análisis de textos literarios. Revista de Filología, Lingüística y Literatura, 32(2), 87-106.

Ribas, Teresa, Milian, Marta, Guasch, Oriol y Camps, Ana. (2002). La composición escrita como objeto de reflexión. En Josep María Cots y Lucila Nussbaum (Eds.), Pensar lo dicho. La reflexión sobre la lengua y la comunicación en el aprendizaje de lenguas (pp. 167-184) Lleida: Milenio.

Sánchez, Carlos. (2005). Los problemas de redacción de los estudiantes costarricenses: una propuesta de revisión desde la lingüística del texto. Revista de Filología y Lingüística, 31(1), 267- 295.

Schön, Donald. (1998). El profesional reflexivo: Cómo piensan los profesionales cuando actúan. Barcelona: Paidós.

Stenhouse, Lawrence. (1987). La investigación como base de la enseñanza. Madrid: Morata.

Tyler, Ralph. (1973). Principios básicos del currículum. Buenos Aires: Troquel.

Van Dijk, Teun A. (1997). Racismo y análisis crítico de los medios. Barcelona: Paidós.

Van Lier, Leo. (2004). Introducing 'language awareness'. London: Penguin.

Walker, Decker. (1990). Fundamentals of currículum. San Diego: Harcourt Brace Jovanovich.

\section{Anexos}

Visualizar los anexos en el siguiente enlace:

https://drive.google.com/file/d/0B6g2xQ4cKGlqeC0zVFIxWkRfV3c/view?usp=sharin

Anexo 1.Tablas descriptivas. Currículum de Español de Costa Rica, séptimo año.

Anexo 2. Redes sistémicas: objetivos, contenidos, procedimientos, valores y actitudes, evaluación de escritura y lectura. Currículum de Español, séptimo año, Costa Rica 